\title{
Ré-émergence de la tuberculose et multirésistance du bacille de Koch
}

\section{Philippe H. Lagrange Alain Wargnier Jean-Louis Herrmann}

P.H. Lagrange, A. Wargnier, J.-L. Herrmann : Service de microbiologie, Hôpital Saint-Louis, 1, avenue Claude Vellefaux, 75475 Paris Cedex 10, France.
La tuberculose aurait dû être en net recul. Malheureusement, il n'en est rien. La progression du nombre des malades est aggravée par la co-infection avec le virus du SIDA, par la désorganisation des systèmes de santé et par l'émergence des bactéries résistantes aux antimycobactériens majeurs. Les progrès récents concernent, d'une part, le génome complet de M. tuberculosis et, d'autre part, les mécanismes physiopathologiques de la maladie, ils ouvrent de nouveaux espoirs vers la maîtrise de cette pandémie mondiale.

\section{TIRÉS À PART}

P.H. Lagrange. ancestrale, reste la maladie infectieuse la plus meurtrière dans le monde. Elle demeure en expansion constante malgré les efforts réalisés pour la contrôler et I'existence depuis trente ans d'une polychimiothérapie antituberculeuse efficace. Près de deux milliards d'individus sont infectés. Le nombre annuel de nouveaux cas est évalué à 8 millions, avec environ 2 millions de décès.

En cette fin de $x x^{\mathrm{e}}$ siècle, la tuberculose reste une maladie étroitement liée à la pauvreté, dramatiquement accentuée dans les pays où les structures de santé sont inexistantes ou déstructurées. Plus de $95 \%$ des cas de tuberculose se retrouvent dans les pays économiquement sous-développés.

Dans ce contexte de pandémie, I'apparition, d'une part, de bacilles tuberculeux multirésistants au traitement, liée à l'absence de prise en charge du traitement correct des patients et, d'autre part, la co-infection par le $\mathrm{VIH}$, qui augmente considérablement le nombre de patients infectés qui évoluent vers une tuberculose maladie contagieuse, crée une situation où tous les éléments sont rassem- blés pour l'apparition d'épidémies de tuberculose grave réfractaire à l'antibiothérapie.

A la fin des années 1970, la mise en place d'une polychimiothérapie obligatoire d'une durée de six mois était acceptée comme l'élément majeur de la lutte contre la tuberculose. On pensait alors que l'éradication était envisageable pour les années 20052010 dans les pays développés comme les États-Unis. Cependant, les événements des deux dernières décennies ont changé dramatiquement la perception, la nature et l'ampleur des problèmes individuels et collectifs posés par la tuberculose. Dès la fin des années 1980, la tuberculose-maladie est reconnue comme demeurant un problème majeur de santé publique au niveau mondial. En 1993, I'Organisation Mondiale de la Santé (OMS) la déclare urgence mondiale.

A partir de 1985 aux États-Unis, la diminution jusqu'alors constante des cas enregistrés s'interrompt conjointement avec la survenue de plusieurs épidémies de tuberculose nosocomiale, responsable d'une grande majorité de décès en raison de l'émergence de souches de Mycobacterium tuberculosis (ou bacilles de 
Koch, BK) multirésistantes (la multirésistance étant définie comme une résistance à l'isoniazide et à la rifampicine), $19 \%$ des souches d'après une étude de Frieden à New York au début des années 1990 [1]. Cependant, le nombre de souches multirésistantes était très inégalement réparti aux États-Unis [1]. De même, au niveau mondial, leur prévalence est très variable, pouvant atteindre des taux très alarmants: 54,4\% en Lettonie [2]. Ces résultats dramatiques sont liés, dans la majorité des cas, à des difficultés opérationnelles majeures dans I'organisation de la lutte contre la tuberculose et sont amplifiés par l'épidémie due à l'infection par le virus de l'immunodéficience humaine (VIH). En effet, la co-infection par le $\mathrm{VIH}$ dans une population très exposée au BK, est un facteur multiplicateur de la morbidité et de la mortalité de la tuberculose, qui par contrecoup, augmente le nombre $d^{\prime}$ isolats multirésistants [3]. C'est dans ce contexte d'échec à l'échelle mondial que se sont développés l'élaboration de nouvelles stratégies par l'OMS et des programmes nationaux et internationaux de recherche visant à améliorer les outils pour combattre avec plus d'efficacité la ré-émergence de cette pandémie mondiale.

\section{La tuberculose dans le monde}

Selon les estimations récentes de I'OMS [4], un tiers de la population mondiale est infecté par le BK, soit deux milliards d'individus qui constituent un réservoir potentiel de bacilles. En 1997, on estime à 16,1 millions le nombre d'invidus ayant une tuberculose active, à 1,9 millions le nombre de décès, à 8 millions le nombre de nouveaux cas dont 3,5 millions qui présentent des bacilles dans leur expectoration et qui sont de ce fait hautement contagieux.

Le nombre de tuberculeux dans le monde est très inégalement réparti. Les pays ayant la plus forte prévalence sont les pays les plus pauvres, où se retrouvent $95 \%$ des tuberculeux et $98 \%$ des décès. Cependant, aucun pays développé n'échappe aux conséquences de cette pandémie mondiale. Ainsi en Europe de l'Ouest, dans le milieu des années
1980-1990, le nombre de cas de tuberculose chez les personnes migrantes, originaires des pays à haute prévalence, a entraîné une inversion de la diminution de l'incidence constamment observée jusqu'alors. En 1997, I'incidence de la tuberculose en France était de $8,1 / 100000$ habitants chez les personnes nées sur le territoire français alors qu'elle était de 44,2/ 100000 habitants pour ceux nés à l'étranger [5]. La disparité entre les pays riches et les pays pauvres devrait encore s'aggraver du fait de la continuelle croissance démographique, de la propagation du VIH, et du manque de moyens engagés pour le contrôle de la maladie.

1. Selon les estimations les moins pessimistes, la population mondiale devrait atteindre 10 milliards dans les prochaines années. Le pourcentage de celle qui vit dans les pays à revenu faible ou intermédiaire a proportionellement beaucoup plus augmenté durant les deux dernières décennies, et cette évolution devrait s'amplifier dans les années à venir. Parallèlement, le développement croissant des concentrations urbaines est à l'évidence source d'une augmentation de la transmission et de la morbidité du fait des mauvaises conditions de vie. 2. La conséquence majeure de l'infection par le $\mathrm{VIH}$ est de multiplier le

nombre de patients qui, infectés par le BK, développeront rapidement une tuberculose-maladie, en tout point aussi contagieuse que la tuberculose survenant chez les patients non infectés par le VIH. Comme le montre le Tableau I, il existe une augmentation considérable du nombre des adultes co-infectés par le VIH et le BK entre 1990 et 1997. Si la majorité de ces personnes sont actuellement en Afrique sub-saharienne, leur nombre s'accroît de façon très importante en Asie du Sud Est [6]. La propagation du VIH sur le continent asiatique est en conséquence potentiellement dramatique concernant la tuberculose, en raison de la prévalence très élevée de l'infection par le BK dans ces populations, la moitié des adultes pouvant être considérée comme réservoirs potentiels. Cette co-infection dans les pays pauvres a créé un problème énorme dans la lutte antituberculeuse comme cela a été mis en évidence dans certains pays d'Afrique où des programmes nationaux parfaitement établis ont été déstabilisés. La Tanzanie est un des exemples dramatiques où I'analyse épidémiologique a montré que l'augmentation régulière des cas de tuberculose était presque complètement liée à la propagation du VIH, passant de 15000 cas déclarés en 1987 à plus de 45000 cas en 1997 [7].

Tableau I. Évolution de la répartition géographique et du nombre de patients estimés co-infectés par M. tuberculosis et le VIH dans la tranche d'âge 15-44 ans (DMS/GTB)

\begin{tabular}{|lrr|}
\hline Région & 1990 & 1997 \\
\hline Afrique Sub Saharienne & 2400000 & 11700000 \\
Amérique du Sud & 301000 & 464000 \\
Asie du Sud Est & 200000 & 2837000 \\
Amérique du Nord & 110000 & 60000 \\
Europe & 48000 & 42000 \\
Russie & 8000 & 23000 \\
Afrique du Nord & 7000 & 75000 \\
Australie, Océanie & 4000 & 1000 \\
Chine $\quad$ ND & 135000 \\
& $\mathbf{3 0 7 8 0 0 0}$ & $\mathbf{1 5 3 7 0 0 0}$ \\
\hline
\end{tabular}


3. La stratégie élaborée, mise en place en 1994 par l'OMS et I'Union Internationale de Lutte contre la Tuberculose et les Maladies Respiratoires (UICTMR), appelée DOTS (Directly Observed Therapy Short Course), c'est-à-dire le contrôle par une tierce personne de la prise effective du traitement quotidien, est normalement adoptée par $50 \%$ des pays membres. Cette méthode a démontré son efficacité pour la prévention des résistances secondaires et primaires dans des pays aussi différents que les États-Unis (ville de New York) et la Guinée $[8,4]$. Cependant, ses taux de couverture varient fortement selon les pays et les régions du monde. Un bilan, fait en 1998, montre que seulement $12 \%$ des cas estimés de tuberculose et $15 \%$ des cas présentant des bacilles à l'examen direct de l'expectoration ont bénéficié $d$ 'une telle approche. Les échecs du traitement restent donc très fréquents et souvent non détectés du fait d'une absence de surveillance des cas traités, ce qui inéluctablement augmente la prévalence de la résistance primaire et secondaire du BK aux antibiotiques. La double résistance à l'isoniazide (INH) et à la rifampicine (RIF) est devenue si préoccupante qu'elle pourrait exiger la détection rapide systématique de la multirésistance et I'adoption d'emblée d'un traitement adapté à cette résistance. La mise en place des programmes nationaux visant à lutter efficacement demande un système de santé publique actif, un personnel entraîné, bien encadré et un investissement financier majeur. Toutes ces conditions n'existent pas dans les pays à forte endémie de tuberculose actuellement, bien que certains pays, comme la Guinée, aient démontré toute l'efficacité d'une telle stratégie.

\section{La multirésistance du BK}

La multirésistance du BK est une définition opérationnelle et correspond à la résistance simultanée à I'INH et à la RIF, deux antituberculeux majeurs. Cette résistance dans le schéma thérapeutique actuellement recommandé diminue de façon certaine l'efficacité du traitement de courte durée (6 mois). L'acquisition d'une souche multirésistance s'effectue soit à la suite d'une infection par un BK d'emblée multirésistant chez un patient n'ayant jamais reçu d'antibiotique auparavant (résistance primaire), soit lorsque le traitement inadéquat ou mal pris, entraîne chez un patient initialement infecté par une souche sensible, une sélection des mutants résistants (résistance acquise ou secondaire). Les antituberculeux ont une activité nette dans le traitement de la tuberculose, mais il fût évident, dès le début de leur utilisation, qu'une monothérapie était responsable d'échecs et de sélection de mutants résistants. Chaque mutation étant indépendante, ce phénomène $a$ été à l'origine de la recommandation obligatoire d'une polychimiothérapie. La résistance acquise, induite par un traitement inadéquat, est donc un marqueur important d'une déficience des programmes de lutte contre la tuberculose [2]. N'ayant pas d'autre alternative thérapeutique, à cause de I'absence du développement d'antituberculeux majeurs équivalents à I'INH et à la RIF, nous nous retrouvons devant une impasse thérapeutique proche de celle d'avant l'ère de I'antibiothérapie. Cette perspective insupportable a engendré une série de décisions stratégiques dont certaines sont actuellement en application. La première a concerné l'analyse épidé- miologique des tuberculoses à BK multirésistants [2], la seconde a été la recommandation d'application généralisée du système DOTS. Les autres, à échéance plus lointaine, ont été de favoriser les recherches, en particulier consacrées à la connaissance du génome de $M$. tuberculosis, ouvrant des perspectives thérapeutiques et physiopathologiques.

En 1994, un projet conjoint de surveillance globale de la multirésistance a été initié par l'OMS et I'UICTMR. Les résultats préliminaires ont été publiés récemment [2]. Globalement, la prévalence moyenne de la résistance secondaire est dix fois supérieure à la résistance primaire (Tableau II). Mais, il existe une disparité très importante suivant les régions, comme cela a été observée par exemple en Europe de l'Ouest en 1997 (Tableau III) [5]. Les résistances primaires sont élevées dans les pays de I'ancienne URSS, en Asie, en République Dominicaine et en Inde (état de New Dehli), révélant des situations potentiellement dramatiques à court et moyen terme. Cette répartition, dominée par les pays à ressources faibles ou intermédiaires, est significative de leur manque de ressources nécessaires à la lutte antituberculeuse.

Tableau II. Exemples de la répartition géographique variable des multirésistances primaires et secondaires en 1997 [2].

\begin{tabular}{|lcc|}
\hline & \multicolumn{2}{c|}{ Pourcentage de souches multirésistantes* } \\
\cline { 2 - 3 } Pays & $\begin{array}{c}\text { Primaire } \\
\text { (Nb de souches testées) }\end{array}$ & $\begin{array}{c}\text { Secondaire } \\
\text { (Nb de souches testées) }\end{array}$ \\
France & $0,5(1491)$ & $4,1(195)$ \\
Russie & $4,0(248)$ & $27,3(33)$ \\
États-Unis & $1,6(3511)$ & $7,1(833)$ \\
Argentine & $4,6(606)$ & $22,2(288)$ \\
Sierra Leone & $1,1(463)$ & $12,8(172)$ \\
Côte d'Ivoire & $5,3(320)$ & - \\
Vietnam & $2,3(640)$ & - \\
République Dominicaine & $6,6(303)$ & $19,9(117)$ \\
Corée & $1,6(2486)$ & $27,5(189)$ \\
\multicolumn{1}{c}{ Total (pays ayant été notifiés) } & $\mathbf{1 , 4}(82)$ & $\mathbf{1 3 , 0}(\mathbf{2 8})$ \\
* Isoniazide et rifampicine résistance & & \\
\hline
\end{tabular}


Tableau III. Multirésistance des souches isolées en Europe chez des patients ayant une tuberculose avec une culture positive en 1997 [5].

\begin{tabular}{|lccr|}
\hline & \multicolumn{3}{c|}{ Nombre de souches multirésistantes $(\%)$} \\
\cline { 2 - 4 } Pays & $\begin{array}{c}\text { Patients jamais traités } \\
\text { (Primaire) }\end{array}$ & $\begin{array}{c}\text { Patients déjà traités } \\
\text { (Secondaire) }\end{array}$ & Total \\
Danemark & $1(0,3)$ & $0(0,0)$ & $1(0,2)$ \\
Estonie & $43(12,9)$ & $6(13,6)$ & $49(13,0)$ \\
Lettonie & $50(14,4)$ & $124(54,4)$ & $174(30,2)$ \\
Islande & $0(0,0)$ & $0(0,0)$ & $0(0,0)$ \\
Hollande & $5(0,6)$ & $2(3,3)$ & $7(0,8)$ \\
Norvège & $1(0,8)$ & $0(0,0)$ & $1(0,8)$ \\
Roumanie & $94(2,8)$ & $37(7,4)$ & $131(3,4)$ \\
Slovénie & $2(0,7)$ & $1(2,9)$ & $3(1,0)$ \\
Suède & $2(0,6)$ & $2(7,7)$ & $4(1,1)$ \\
Suisse & $0(0,0)$ & $5(11,9)$ & $5(1,1)$ \\
\end{tabular}

La mobilisation des moyens de lutte, avec une mise en application du système DOTS la plus généralisée possible, ne peut se concevoir que de façon globale et mondialiste. Elle nécessite une implication commune des pays pauvres et des pays riches, mutualisant leurs moyens afin de coordonner rapidement les outils actuels et ceux issus de la recherche.

\section{La recherche fondamentale et appliquée}

La connaissance de la séquence complète du génome de $M$. tuberculosis H37Rv (souche pathogène de référence) est certainement une avancée majeure capable de promouvoir à terme la conception de nouvelles thérapeutiques et de nouveaux vaccins par une meilleure appréhension des mécanismes moléculaires de la pathogénicité du BK [10]. La publication de cette séquence complète [11], la mise au point d'outils de recherche permettant I'analyse de génétique moléculaire de M. tuberculosis [12, 13], la génomique comparative des différentes souches appartenant au complexe tuberculosis, dont $M$. bovis, et la souche vaccinale atténuée (M. bovis, BCG), permettront une meilleure compréhension des bases moléculaires de la pathogénicité [14,
M. tuberculosis [17]. L'importance de certains locus pour la viabilité du BK chez l'animal, démontrée récemment par mutagénèse par transposition, implique des gènes codant pour le métabolisme lipidique [13].

Les données obtenues par visualisation directe en microscopie électronique des relations des mycobactéries avec les membranes cellulaires montrent à l'évidence la nécessité d'un contact intime associé à la survie des bactéries [18].

Enfin, il faut signaler la mise en évidence récente de l'importance des antigènes non protéiques dérivés des lipides de la paroi (lipoarabinomananes et acides mycoliques) qui sont reconnus par des cellules lymphocytaires $\mathrm{T}$ cytotoxiques $\mathrm{CD}^{+}$et doublement négatives (CD4-, CD8-) par l'intermédiaire $d^{\prime}$ une présentation de l'antigène par des molécules CD1, indépendantes du complexe majeur d'histocompatibilité. Ces cellules ont une activité cytotoxique mais aussi directement antimycobactérienne par I'intermédiaire d'un polypeptide cationique appelé granulysine [19].

Néanmoins, l'intégration de ces données concernant le rôle respectif des différentes classes de lipides, celui des cellules lymphocytaires, celui des cellules présentatrices de I'antigène pour l'induction (les cellules dendritiques) ou pour l'expression (les macrophages) des réponses immunitaires n'est pas encore réalisée. Elle doit être envisagée en priorité pour mieux appréhender leurs rôles respectifs dans la mise en place des éléments constitutifs de la protection spontanée, de l'immunité adaptative, et ceux impliqués dans les conditions pathologiques associées aux différentes étapes de la tuberculose-maladie. De nombreuses évidences tendent à montrer que les conditions conduisant à l'expression clinique, radiologique et biologique de la tuberculose résultent des réponses inflammatoires et immunologiques excessives développées par l'hôte en contact avec les antigènes mycobactériens [20].

D'autres retombées potentielles des recherches actuellement menées concernent le développement et la validation d'outils pour un meilleur diagnostic de la maladie. Ils complétent I'arsenal actuel, et mettent en évidence des constituants nucléosi- 
diques, biochimiques et antigéniques du BK grâce au développement de techniques issues de la biologie moléculaire et de l'immunologie.

Il sera également important de développer et valider les outils cellulaires ou moléculaires permettant d'analyser les réponses immunitaires se développant après l'infection, en différenciant celles associées à la protection de celles impliquées dans l'immunopathologie. A côté des facteurs de risque influençant la transmission du BK, une meilleure connaissance des facteurs individuels qui conduisent à la maladie permettra une meilleure prise en charge "prophylactique» ciblée des patients infectés, à haut risque de développer une tuberculose-maladie. Enfin, les progrès thérapeutiques attendus seront issus de la connaissance des bases moléculaires des différentes voies métaboliques qui régissent la multiplication et la persistance des mycobactéries en position intracellulaire chez l'hôte infecté. De même, il est vraisemblable que les connaissances acquises sur le rôle des réponses inflammatoires et immunopathologiques de l'hôte, serviront à envisager l'impact des substances adjuvantes dans la modulation de ces réponses soit dans la prévention, soit dans l'accélération de la guérison sous l'influence des thérapeutiques strictement antimycobactériennes

\section{RÉFÉRENCES}

1. Frieden TR, Sterling T, Pablos-Mendez A Kilburn JD, Cauthen GM, Dooley SW. The emergence of drug resistant tuberculosis in New york City. N Engl J Med 1993; 328: $521-8$.

2. Pablos-Mendez A, Raviglione MC, Lazlo A et al. Global surveillance for antituberculosis resistance 1994-1997. N Engl / Med $1998 ; 338: 1641-9$

3. Narain JP, Raviglione MC, Kochi A. HIVassociated tuberculosis in developping countries: epidemiology and strategies for prevention. Tuber Lung Dis 1992; 73: 311-21.

4. World Health Organization. Global tuberculosis control. WHO report 1999. Geneva, Switzerland: WHO/CDS/CPC/TB 1999: 99-259.

5. Euro TB (CESES/KNCV) and the national coordinators for tuberculosis surveillance in the WHO European region. Surveillance of tuberculosis in Europe. Report on tuberculosis cases notified in 1997. EuroTB 1999:1-95.
6. Murray JF. Tuberculosis and HIV infection. A global prespective. Respiration $1998 ; 65: 335-42$

7. Van Cleef MRA, Chum HJ. The proportion of Tuberculosis in Tanzania attributable to Human Immunodeficiency Virus. Int $J$ Epidemiol 1995; 24 : 637-42.

8. Frieden TR, Fujiwara PI, Washko RM, Hamburg M. Tuberculosis in New York City-turning the tide. N Engl J Med 1995; 333: 229-33.

9. Grosset J. Quelle(s) stratégie(s) pour l'éradication de la tuberculose. Bull Acad Nat Med 1999; 183: 25-37.

10. Cole ST. Comparative mycobacterial genomics. Curr Opin Microbiol 1998; 1 : 567-71.

11. Cole ST, Brosch R, Parkhill J, et al. Deciphering the biology of Mycobacterium tuberculosis from complete genome sequence. Nature 1998; 393: 537-44.

12. Pelicic $V$, Jackson $M$, Peyrat JM, Jacobs WR, Gicquel B. Efficient allelic exchange and transposon mutagenesis in Mycobacterium tuberculosis. Proc Natl Acad Sci USA 1997; 94 : 10955-60.

13. Camacho LR, Ensergueix D, Perez E, Gicquel B, Guilhoz C. Identification of virulence gene cluster of Mycobacterium tuberculosis by signature-tagged transposon mutagenesis. Mol Microbiol 1999; 34 : 257 67.

14. Behr M, Wilson M, Gill W, et al. Comparative genomics of $\mathrm{BCG}$ vaccines by whole-genome DNA microarray. Science 1999 : 284: 1520-3.

15. Gordon S, Brosch R, Billault A, Garnier $\mathrm{T}$, Eiglmeier $\mathrm{K}$, Cole $\mathrm{S}$. Identification of variable regions in the genomes of tubercle bacilli using bacterial artificial chromosome arrays. Mol Microbiol 1999; 32 : 643-655.

16. Lagrange P.H. Nouvelles connaissances sur la structure des mycobactéries. Rev Pneumol Clin 1994; 50:199-206.

17. Ferrari G, Langen H, Naito M, Pieters J. A coat protein on phagosomes involved in the intracellular survival of mycobacteria. Cell 1999; 97: 435-47.

18. Molloy A, Laochumroonvorapong $\mathrm{P}$. Kaplan G. Apoptosis but not necrosis of infected monocytes is compled with killing of intracellular Bacillus Calmette-Guerin. Exp Med 1994; 180: 1499-509.

19. Stenger S, Mazzaccaro RJ, Uyemura K et al. Differential effects of cytolytic T cell subsets on intracellular infection. Science 1997 ; 276 : 1684-7.

20. Lagrange PH, Wargnier A, Herrmann JL. The immune responses in tuberculosis: role for pathogenesis, diagnosis and prevention. Pediatr Pulmonol 1999; 18: 136-9.

\section{$\mathbf{m} \mathbf{S} 2000$ \\ Summary}

Tuberculosis, a reemergent

disease

Tuberculosis, an ancestral human disease, remains the most widespread infectious disease in the world. Tuberculosis remains in constant expansion and the number of new cases is projected to continue to rise, especially in lowincome countries in spite of all efforts made for its eradication and the existing efficacious treatment, known for more than 30 years. About one third of the world population (2 billions) is already infected with Mycobacterium tuberculosis. The annual number of new cases of tuberculosis is estimated to about 8 millions of patients, with 2 millions of deaths. At the end of the XXth century, tuberculosis remains a disease closely related to poverty and is dramatically more marked in countries or regions where the tuberculosiscontrol programs are absent or poorly functionning. More than $95 \%$ of all tuberculosis cases are present in the low-income countries. Within this pandemic context, the emergence of multidrug resistant strains, on the one hand, arising mostly after inadequate treatment and follow-up, and on the other hand, the HIV coinfection that increases considerably the number of tuberculosis infected individuals moving rapidly to contagious tuberculosis disease, create new conditions where all the constituants are assembled for the occurence of outbreaks of severe tuberculosis unmanageable by current antimycobacterial antibiotics. Joint efforts should address the issue of the global implantation of the World Health Organisation recommandations to control tuberculosis and of research needs for developing new effective vaccine and new drugs based upon new knowledge on molecular and cellular physiopathological mechanisms of tubercle bacillus in the host. 